\title{
ESTIMATION OF AR(1) PARAMETER WITH CONFIDENCE
}

\section{SHIPRA BANIK and B. M. GOLAM KIBRIA}

Department of Physical Sciences

Independent University, Bangladesh

Dhaka

Bangladesh

e-mail: banik@iub.edu.bd

Department of Mathematics and Statistics

Florida International University

University Park, Miami FL 33199

USA

e-mail: kibriag@fiu.edu

\begin{abstract}
This paper considers some bootstrap version of the existing confidence intervals for estimating the parameter of an autoregressive process of order one model. A simulation study has been conducted to compare the performance of the proposed intervals using two important measures: coverage probability and average width. It appears from our simulation study that all methods have confidence coefficient closest to the given confidence coefficient, however, our proposed bootstrap intervals have small average widths as compare to its counterpart. A real life data are analyzed, which supported the simulation results to some extent. We believe that the findings of this study will make important contribution to the time series literature.
\end{abstract}

2010 Mathematics Subject Classification: Primary 62F10; Secondary 62F99.

Keywords and phrases: bootstrapping, confidence intervals, simulation, stock price index, time series models.

Received October 21, 2018

(ㄷ) 2019 Scientific Advances Publishers 


\section{Introduction}

A time series is an ordered sequence of values of a variable that occurred at equally spaced points in time. It is well known that the time series analysis comprises methods for analyzing time series data in order to extract meaningful statistics and other characteristics of a data set. Another very widely used area known as time series forecasting or predicting of future values based on observed data. To predict any series of data, one might need to know about the values of population parameter of the model. Since the parameters are unknown, it is essential to estimate them from the observed data. To estimate parameters, there are two methods available in literature: (i) point estimation, (ii) confidence interval estimation. Since point estimator is not reliable, one can rely on the confidence interval method, which will capture the true population parameter in repeated samples with some confidence. Recently, confidence interval for an AR(1) time series model has been given considerable attention in the literature. Fuller [5] initiated to construct a confidence interval of an AR(1) parameter based on the AR coefficient, which is estimated by the ordinary least squares (OLS) method. Evidences (Dickey and Fuller [2] and others) show that the limiting distribution of this $t$-statistic based on the OLS method varies on different values of $\mathrm{AR}(1)$ parameter values. To overcome this noise, So and Shin [1] for an AR(1) model propose a new confidence interval estimators based on the Cauchy estimator, whose statistic have the standard normal limiting distribution for all ranges of the AR parameters values. Their Monte-Carlo simulations for an AR(1) process shows that the proposed test is more powerful than the Fuller [5] test. It also shows that So and Shin [1] proposed confidence interval has shorter average lengths than the Fuller [5] suggested interval. Phillips et al. [7] suggested a better new simple confidence interval for an AR(1) parameter as compare to Beong and Dong [1] interval based on an instrumental estimator, which has better finite sample properties according to Phillips et al. [7] simulation studies. Gallagher and Tunno [6] proposed an interval for an $\mathrm{AR}(1)$ parameter for small samples to improve finite sample properties by combining the OLS estimator and the Cauchy 
estimator. Their simulation evidences show that the suggested interval has shorter average width as compare to the Beong and Dong [1] interval and the Phillips et al. [7] interval. To improve finite sample properties, recently, Ferebee and Ashton [4] constructed an interval by combining the weighted least square estimator and the sample autocorrelation function of lag one. Their simulation results show that their proposed interval has better finite sample properties as compare to the proposed Fuller [5] interval. To improve finite sample performance one can typically use bootstrapping, which is a frequently used computer-based non-parametric procedure familiarized by Efron [3]. It is a technique, which necessitates no assumptions regarding the underlying target population and can be applied to a range of circumstances. The bootstrapping confidence interval of an $\mathrm{AR}(1)$ model in context of time series analysis is limited in literature. The purpose of this paper is to propose some bootstrap versions of confidence interval for an AR(1) process and is to compare their performances to the existing intervals by a simulation study. The structure of the paper is as follows: We review and propose some confidence intervals for an AR(1) model in Section 2. Simulation study with results is discussed in the Section 3. Real life application has been given in the Section 4 and Section 5 concludes the paper.

\section{Existing and Proposed Confidence Intervals (CI) for an AR(1) Model}

Consider the following $\mathrm{AR}(1)$ process:

$$
y_{t}=\rho y_{t-1}+e_{t}, t=1,2, \ldots, n,
$$

where $y_{t}$ is a time series data and $e_{t}$ is a sequence of independent and identically distributed (i.i.d.) errors having zero mean and finite variance $\sigma^{2}$. The available existing intervals and our proposed bootstrap versions intervals are briefly explained in this section. 


\subsection{Fuller CI}

According to Fuller [5], the $100(1-\alpha) \% C I$ for $\rho$ is given by

$$
L C L=\hat{\rho}-z \alpha / 2 s e(\hat{\rho}) \text { and } U C L=\hat{\rho}+z \alpha / 2 s e(\hat{\rho}),
$$

where $\hat{\rho}=\frac{\sum_{t=1}^{n} y_{t-1} y_{t}}{\sum_{t=1}^{n} y_{t-1}^{2}}, s e(\hat{\rho})$ is the standard error of $\hat{\rho}$ and $z \alpha / 2$ is the standard normal quantile with an area of $\alpha / 2$ to its right.

\subsection{Beong and Dong CI}

Beong and Dong [1] proposed an interval based on the Cauchy estimator (has certain advantages over the proposed Fuller [5] confidence interval). Their proposed $100(1-\alpha) \% C I$ for $\rho$ is defined as

$$
L C L=\hat{\rho}_{c}-z_{\alpha / 2} \operatorname{se}\left(\hat{\rho}_{c}\right) \text { and } U C L=L C L=\hat{\rho}_{c}+z_{\alpha / 2} s e\left(\hat{\rho}_{c}\right),
$$

where

$$
\hat{\rho}_{c}=\frac{\sum_{t=2}^{n} s_{t-1} y_{t}}{\sum_{t=2}^{n}\left|y_{t-1}\right|}, S_{t}=\left\{\begin{array}{l}
1, X_{t}>0 \\
0, X_{t}=0 \\
-1, X_{t}<0
\end{array}, \operatorname{se}\left(\hat{\rho}_{c}\right)=n^{0.5} \hat{\sigma} / \sum_{t=2}^{n}\left|y_{t-1}\right|\right.
$$

$\hat{\sigma}=\sqrt{\sum_{t=2}^{n}\left(y_{t}-\hat{\rho}_{0} y_{t-1}\right)^{2} /(n-2)}, \hat{\rho}$ is the OLS estimator and $z_{\alpha / 2}$ is the standard normal quantile with an area of $\alpha / 2$ to its right.

\subsection{Philips et al. CI}

Phillips et al. [7] suggested an interval based on the Cauchy estimator and showed that this estimator has asymptotically optimal precision properties in a certain class of instrumental variable estimators. Their proposed $100(1-\alpha) \% C I$ for $\rho$ is defined as

$$
L C L=\hat{\rho}_{c}-z_{\alpha / 2} \operatorname{se}\left(\hat{\rho}_{c}\right) \text { and } U C L=L C L=\hat{\rho}_{c}+z_{\alpha / 2} s e\left(\hat{\rho}_{c}\right) \text {, }
$$


where $\operatorname{se}\left(\hat{\rho}_{c}\right)=\hat{\sigma} \frac{\sqrt{\sum_{t=2}^{n} s_{t-1}^{2}}}{\sum_{t=2}^{n}\left|y_{t-1}\right|}, \hat{\rho}_{c}$ is defined above and $z_{\alpha / 2}$ is the standard normal quantile with an area of $\alpha / 2$ to its right.

\subsection{Gallagher and Tunno CI}

Gallagher and Tunno [6] proposed an interval, which is a small sample correction of the intervals suggested by So and Shin [1] and Phillips et al. [7]. They constructed an interval for $\rho$ around a linear combination of the OLS estimator and the Cauchy estimator. Their proposed $100(1-\alpha) \% C I$ for $\rho$ is defined as

$$
L C L=\hat{\rho}-z \alpha_{/ 2} \sqrt{\operatorname{Var}(\hat{\rho})} \text { and } U C L=\hat{\rho}+z \alpha / 2 \sqrt{\operatorname{Var}(\hat{\rho})},
$$

where $\sqrt{\operatorname{Var}(\hat{\rho})}=\frac{\sigma^{2} \sum_{t=2}^{n} y_{t-1}^{2}}{\left(n^{0.5} \sum_{t=2}^{n}\left|y_{t-1}\right|^{2}\right)^{2}}$ and $z_{\alpha / 2}$ is the standard normal quantile with an area of $\alpha / 2$ to its right.

\subsection{Ferebee and Ashton CI}

To improve coverage probability, Ferebee and Ashton [4] proposed interval based on a linear combination of the Cauchy estimator and the sample autocorrelation function of lag one. The $100(1-\alpha) \% C I$ for $\rho$ is given by

$$
L C L=\hat{p}(1)-z \alpha / 2 \sqrt{V(\hat{p}(1))} \text { and } U C L=\hat{p}(1)+z \alpha / 2 \sqrt{V(\hat{p}(1))},
$$

where $\hat{\rho}(1)=\frac{\sum_{t=2}^{n} y_{t-1} y_{t}}{\sum_{t=1}^{n} y_{t}^{2}}, V(\hat{p}(1))=\frac{\sigma^{2}}{\sum_{t=1}^{n} y_{t}^{2}}, \sigma^{2}$ is the variance of the error process and $z_{\alpha / 2}$ is the standard normal quantile with an area of $\alpha / 2$ to its right. 


\subsection{Proposed Bootstrap CIs}

Let $X^{\left({ }^{*}\right)}=X_{1}^{\left(*^{*}\right)}, X_{2}^{\left({ }^{*}\right)}, \ldots, X_{n}^{(*)}$, where the $i$-th sample is denoted $X^{(i)}$ for $i=1,2, \ldots, B$ and $B$ is the number of bootstrap samples. The bootstrap estimate of $\rho$ is $\rho_{i}^{*}$ for $n$ estimates. We proposed the following bootstrap version confidence intervals of $\rho$ :

\subsubsection{Bootstrap Fuller (BF) CI}

The $100(1-\alpha) \% C I$ for $\rho$ is given by

$$
L C L=\widehat{\rho}-z_{\alpha / 2}^{*} s e(\hat{\rho}) \text { and } U C L=\widehat{\rho}+z_{\alpha / 2}^{*} s e(\widehat{\rho}),
$$

where $z_{\alpha / 2}^{*}$ is the $(\alpha / 2)$-th quintile of the $z$-statistic.

\subsubsection{Bootstrap Beong and Dong (BSS) CI}

The $100(1-\alpha) \% C I$ for $\rho$ is given by

$$
L C L=\hat{\rho}_{c}-z_{\alpha / 2}^{*} s e\left(\hat{\rho}_{c}\right) \text { and } U C L=L C L=\widehat{\rho}_{c}+z_{\alpha / 2}^{*} s e\left(\hat{\rho}_{c}\right),
$$

where $z_{\alpha / 2}^{*}$ is the $(\alpha / 2)$-th quintile of the $z$-statistic.

\subsubsection{Bootstrap Phillips et al. (BP) CI}

The $100(1-\alpha) \% C I$ for $\rho$ is given by

$$
L C L=\widehat{\rho}_{c}-z_{\alpha / 2}^{*} \operatorname{se}\left(\hat{\rho}_{c}\right) \text { and } U C L=L C L=\widehat{\rho}_{c}+z_{\alpha / 2}^{*} s e\left(\widehat{\rho}_{c}\right) \text {, }
$$

where $z_{\alpha / 2}^{*}$ is the $(\alpha / 2)$-th quintile of the $z$-statistic.

\subsubsection{Bootstrap Gallagher and Tunno (BGT) CI}

The $100(1-\alpha) \% C I$ for $\rho$ is given by

$$
L C L=\hat{\rho}-z_{\alpha / 2}^{*} \sqrt{\operatorname{Var}(\hat{\rho})} \text { and } U C L=\hat{\rho}+z_{\alpha / 2}^{*} \sqrt{\operatorname{Var}(\hat{\rho})},
$$

where $z_{\alpha / 2}^{*}$ is the $(\alpha / 2)$-th quintile of the $z$-statistic. 


\subsubsection{Bootstrap Ferebee and Ashton (BTE) CI}

The $100(1-\alpha) \% C I$ for $\rho$ is given by

$$
L C L=\hat{p}(1)-z_{\alpha / 2}^{*} \sqrt{V(\hat{p}(1))} \text { and } U C L=\hat{p}(1)+z_{\alpha / 2}^{*} \sqrt{V(\hat{p}(1))},
$$

where $z_{\alpha / 2}^{*}$ is the $(\alpha / 2)$-th quintile of the $z$-statistic.

\section{Simulation Study}

Since a theoretical comparison among the intervals is not possible, a simulation study has been conducted in this paper to find some good intervals based on calculated coverage probability and average length width of selected intervals. The design of our study is as follows:

\subsection{Simulation design}

Since, random samples are an important component for estimating parameter, we have varied sample sizes as follows: $n=20$, 50, and 100 . For each $n$, random samples are generated from an AR(1) process with normal error distribution and various parameter values of $\rho=-0.9,-0.8,-0.7,-0.5,0.5,0.6,0.8$, and 0.9 to see effects of $\rho$ on our considered confidence intervals. 5000 replications are used for each case and 2500 bootstrap samples for each $n$. The most common $95 \%$ confidence interval for the confidence coefficient is used. In order to compare performance of the various intervals, the following criterions are considered: (i) coverage probability and (ii) mean width on the resulting confidence intervals. Simulation results for various values of $\rho$ are tabulated for selected values of $n$ in Tables 3.1 to 3.3, respectively. 
Table 3.1. Coverage probability and average width of selected intervals when data generated from an $\mathrm{AR}(1)$ model with for various values of $\mathrm{AR}$ coefficients and $n=20$

\begin{tabular}{|c|c|c|c|c|c|c|c|c|}
\hline \multicolumn{9}{|c|}{ AR(1) parameter values } \\
\hline Intervals & $\rho=-0.9$ & $\rho=-0.8$ & $\rho=-0.7$ & $\rho=-0.5$ & $\rho=0.5$ & $\rho=0.6$ & $\rho=0.8$ & $\rho=0.9$ \\
\hline \multirow[t]{2}{*}{ OLS } & 0.9425 & 0.9433 & 0.9434 & 0.9436 & 0.9417 & 0.9425 & 0.9378 & 0.9394 \\
\hline & 3.6818 & 3.7039 & 3.7309 & 3.7502 & 3.7531 & 3.7454 & 3.7073 & 3.6800 \\
\hline \multirow[t]{2}{*}{ Beong and Dong } & 0.9628 & 0.9510 & 0.9489 & 0.9442 & 0.9458 & 0.9425 & 0.9500 & 0.9615 \\
\hline & 0.5988 & 0.7276 & 0.8305 & 0.9796 & 0.9784 & 0.9148 & 0.7305 & 0.5999 \\
\hline \multirow[t]{2}{*}{ Phillips et al. } & 0.9600 & 0.9453 & 0.9431 & 0.9386 & 0.9378 & 0.9365 & 0.9437 & 0.9557 \\
\hline & 0.5837 & 0.7092 & 0.8095 & 0.9548 & 0.9537 & 0.8917 & 0.7120 & 0.5847 \\
\hline \multirow[t]{2}{*}{ Gallagher and Tunno } & 0.9245 & 0.8996 & 0.8931 & 0.8748 & 0.8751 & 0.8754 & 0.8995 & 0.9196 \\
\hline & 0.4974 & 0.5932 & 0.6692 & 0.7765 & 0.7762 & 0.7306 & 0.5960 & 0.4986 \\
\hline \multirow[t]{2}{*}{ Tunno and Erwin } & 0.9227 & 0.9372 & 0.9383 & 0.9401 & 0.9402 & 0.9420 & 0.9306 & 0.9175 \\
\hline & 0.4801 & 0.5753 & 0.6498 & 0.7561 & 0.7547 & 0.7100 & 0.5776 & 0.4812 \\
\hline \multirow[t]{2}{*}{ Bootstrap OLS } & 0.9373 & 0.9410 & 0.9401 & 0.9407 & 0.9406 & 0.9587 & 0.9548 & 0.9317 \\
\hline & 3.5847 & 3.6377 & 3.6919 & 3.7244 & 3.7428 & 3.7435 & 3.6626 & 3.6357 \\
\hline \multirow[t]{2}{*}{ Bootstrap Beong and Dong } & 0.9596 & 0.9475 & 0.9463 & 0.9431 & 0.9450 & 0.9325 & 0.9457 & 0.9548 \\
\hline & 0.5830 & 0.7146 & 0.8218 & 0.9728 & 0.9757 & 0.9144 & 0.7217 & 0.5927 \\
\hline \multirow[t]{2}{*}{ Bootstrap Phillps et al. } & 0.9552 & 0.9402 & 0.9402 & 0.9366 & 0.9368 & 0.9252 & 0.9398 & 0.9500 \\
\hline & 0.5683 & 0.6965 & 0.8010 & 0.9482 & 0.9510 & 0.8912 & 0.7034 & 0.5776 \\
\hline \multirow[t]{2}{*}{ Bootstrap Gallagher and Tunno } & 0.9151 & 0.8937 & 0.8886 & 0.8708 & 0.8734 & 0.8649 & 0.8913 & 0.9118 \\
\hline & 0.4843 & 0.5826 & 0.6622 & 0.7712 & 0.7741 & 0.7302 & 0.5888 & 0.4926 \\
\hline \multirow[t]{2}{*}{ Bootstrap Tunno and Erwin } & 0.9134 & 0.9315 & 0.9345 & 0.9380 & 0.9387 & 0.9436 & 0.9474 & 0.9118 \\
\hline & 0.4675 & 0.5650 & 0.6430 & 0.7509 & 0.7526 & 0.7096 & 0.5706 & 0.4926 \\
\hline
\end{tabular}


Table 3.2. Coverage probability and average width of selected intervals when data generated from an $\mathrm{AR}(1)$ model with for various values of $\mathrm{AR}$ coefficients and $n=50$

\begin{tabular}{|c|c|c|c|c|c|c|c|c|}
\hline \multicolumn{9}{|c|}{ AR(1) parameter values } \\
\hline Selected Intervals & $\rho=-0.9$ & $\rho=-0.8$ & $\rho=-0.7$ & $\rho=-0.5$ & $\rho=0.5$ & $\rho=0.6$ & $\rho=0.8$ & $\rho=0.9$ \\
\hline \multirow[t]{2}{*}{ OLS } & 0.9788 & 0.9757 & 0.9699 & 0.9569 & 0.9584 & 0.9638 & 0.9738 & 0.9786 \\
\hline & 3.8246 & 3.8346 & 3.8370 & 3.8569 & 3.8500 & 3.8452 & 3.8326 & 3.8230 \\
\hline \multirow[t]{2}{*}{ So and Shin } & 0.9554 & 0.9505 & 0.9458 & 0.9465 & 0.9462 & 0.9449 & 0.9533 & 0.9538 \\
\hline & 0.3368 & 0.4352 & 0.5074 & 0.6091 & 0.6095 & 0.5646 & 0.4357 & 0.3381 \\
\hline \multirow[t]{2}{*}{ Phillips et al. } & 0.9535 & 0.9476 & 0.9435 & 0.9441 & 0.9436 & 0.9424 & 0.9515 & 0.9513 \\
\hline & 0.3334 & 0.4308 & 0.5023 & 0.6029 & 0.6033 & 0.5589 & 0.4313 & 0.3347 \\
\hline \multirow[t]{2}{*}{ Gallagher and Tunno } & 0.9025 & 0.8877 & 0.8794 & 0.8808 & 0.8808 & 0.8740 & 0.8959 & 0.9014 \\
\hline & 0.2753 & 0.3516 & 0.4070 & 0.4851 & 0.4855 & 0.4511 & 0.3519 & 0.2766 \\
\hline \multirow[t]{2}{*}{ Tunno and Erwin } & 0.9388 & 0.9446 & 0.9465 & 0.9458 & 0.9479 & 0.9450 & 0.9434 & 0.9378 \\
\hline & 0.2719 & 0.3476 & 0.4026 & 0.4802 & 0.4804 & 0.4464 & 0.3479 & 0.2731 \\
\hline \multirow[t]{2}{*}{ Bootstrap OLS } & 0.9780 & 0.9752 & 0.9729 & 0.9575 & 0.9574 & 0.9641 & 0.9790 & 0.9826 \\
\hline & 3.7681 & 3.7886 & 3.8726 & 3.8222 & 3.8289 & 3.8346 & 3.8200 & 3.7379 \\
\hline \multirow[t]{2}{*}{ Bootstrap So and Shin } & 0.9525 & 0.9472 & 0.9483 & 0.9449 & 0.9447 & 0.9443 & 0.9525 & 0.9502 \\
\hline & 0.3319 & 0.4299 & 0.5121 & 0.6036 & 0.6061 & 0.5630 & 0.4342 & 0.3305 \\
\hline \multirow[t]{2}{*}{ Bootstrap Phillips et al. } & 0.9503 & 0.9443 & 0.9460 & 0.9431 & 0.9424 & 0.9416 & 0.9503 & 0.9481 \\
\hline & 0.3285 & 0.4256 & 0.5070 & 0.5975 & 0.6000 & 0.5574 & 0.4299 & 0.3272 \\
\hline \multirow[t]{2}{*}{ Bootstrap Gallagher and Tunno } & 0.8972 & 0.8836 & 0.8826 & 0.8752 & 0.8786 & 0.8729 & 0.8936 & 0.8957 \\
\hline & 0.2712 & 0.3474 & 0.4108 & 0.4817 & 0.4828 & 0.4499 & 0.3508 & 0.2704 \\
\hline \multirow[t]{2}{*}{ Bootstrap Tunno and Erwin } & 0.9347 & 0.9428 & 0.9495 & 0.9416 & 0.9452 & 0.9453 & 0.9501 & 0.9456 \\
\hline & 0.2679 & 0.3435 & 0.4064 & 0.4767 & 0.4778 & 0.4451 & 0.3468 & 0.2670 \\
\hline
\end{tabular}


Table 3.3. Coverage probability and average width of selected intervals when data generated from an $\mathrm{AR}(1)$ model with for various values of $\mathrm{AR}$ coefficients and $n=100$

\begin{tabular}{|c|c|c|c|c|c|c|c|c|}
\hline \multicolumn{9}{|c|}{ AR(1) parameter values } \\
\hline Selected Intervals & $\rho=-0.9$ & $\rho=-0.8$ & $\rho=-0.7$ & $\rho=-0.5$ & $\rho=0.5$ & $\rho=0.6$ & $\rho=0.8$ & $\rho=0.9$ \\
\hline \multirow[t]{2}{*}{ OLS } & 0.9906 & 0.9868 & 0.9780 & 0.9684 & 0.9695 & 0.9745 & 0.9857 & 0.9940 \\
\hline & 3.8704 & 3.8780 & 3.8816 & 3.8828 & 3.8858 & 3.8818 & 3.8772 & 3.8716 \\
\hline \multirow[t]{2}{*}{ So and Shin } & 0.9495 & 0.9465 & 0.9501 & 0.9475 & 0.9458 & 0.9469 & 0.9491 & 0.9511 \\
\hline & 0.2270 & 0.3011 & 0.3557 & 0.4282 & 0.4277 & 0.3960 & 0.3016 & 0.2261 \\
\hline \multirow[t]{2}{*}{ Phillips et al. } & 0.9483 & 0.9456 & 0.9488 & 0.9458 & 0.9450 & 0.9462 & 0.9480 & 0.9501 \\
\hline & 0.2259 & 0.2996 & 0.3540 & 0.4261 & 0.4256 & 0.3940 & 0.3001 & 0.2250 \\
\hline \multirow[t]{2}{*}{ Gallagher and Tunno } & 0.8888 & 0.8802 & 0.8867 & 0.8790 & 0.8758 & 0.8767 & 0.8860 & 0.8940 \\
\hline & 0.1839 & 0.2420 & 0.2847 & 0.3415 & 0.3411 & 0.3163 & 0.2425 & 0.1832 \\
\hline \multirow[t]{2}{*}{ Tunno and Erwin } & 0.9404 & 0.9465 & 0.9463 & 0.9481 & 0.9473 & 0.9463 & 0.9484 & 0.9444 \\
\hline & 0.1828 & 0.2407 & 0.2832 & 0.3397 & 0.3393 & 0.3147 & 0.2412 & 0.1822 \\
\hline \multirow[t]{2}{*}{ Bootstrap OLS } & 0.9907 & 0.9868 & 0.9772 & 0.9708 & 0.9680 & 0.9778 & 0.9862 & 0.9943 \\
\hline & 3.8540 & 3.8725 & 3.8942 & 3.8932 & 3.8873 & 3.9667 & 3.8539 & 3.9053 \\
\hline \multirow[t]{2}{*}{ Bootstrap So and Shin } & 0.9482 & 0.9465 & 0.9502 & 0.9478 & 0.9449 & 0.9506 & 0.9470 & 0.9537 \\
\hline & 0.2261 & 0.3007 & 0.3569 & 0.4294 & 0.4279 & 0.4046 & 0.2998 & 0.2281 \\
\hline \multirow[t]{2}{*}{ Bootstrap Phillips et al. } & 0.9475 & 0.9455 & 0.9493 & 0.9466 & 0.9435 & 0.9494 & 0.9458 & 0.9525 \\
\hline & 0.2249 & 0.2992 & 0.3551 & 0.4272 & 0.4257 & 0.4026 & 0.2983 & 0.2269 \\
\hline \multirow[t]{2}{*}{ Bootstrap Gallagher and Tunno } & 0.8877 & 0.8801 & 0.8876 & 0.8802 & 0.8754 & 0.8863 & 0.8835 & 0.8976 \\
\hline & 0.1831 & 0.2417 & 0.2856 & 0.3424 & 0.3412 & 0.3232 & 0.2410 & 0.1848 \\
\hline \multirow[t]{2}{*}{ Bootstrap Tunno and Erwin } & 0.9403 & 0.9463 & 0.9467 & 0.9505 & 0.9470 & 0.9508 & 0.9487 & 0.9483 \\
\hline & 0.1820 & 0.2404 & 0.2842 & 0.3406 & 0.3395 & 0.3216 & 0.2397 & 0.1837 \\
\hline
\end{tabular}




\subsection{Results discussion}

For a better presentation, the coverage probability and mean width are presented in Figures 3.1(a) and 3.1(b), respectively. From the Figure 3.1(a), it is evident that for strong correlation (negative and positive) coefficients, coverage probabilities for all intervals but the Gallagher and Tunno interval and the Tunno and Erwin interval are very close to the nominal level. Overall, all intervals performed well in terms of coverage probability except the Gallagher and Tunno interval and also its bootstrap version interval. These two versions coverage probabilities observed below the nominal level. 


$$
\begin{aligned}
& r=-0.9 \square r=-0.8 \square r=-0.7 \square r=-0.5 \\
& \square=0.5 \square r=0.6 \square r=0.8 \backsim r=0.9
\end{aligned}
$$

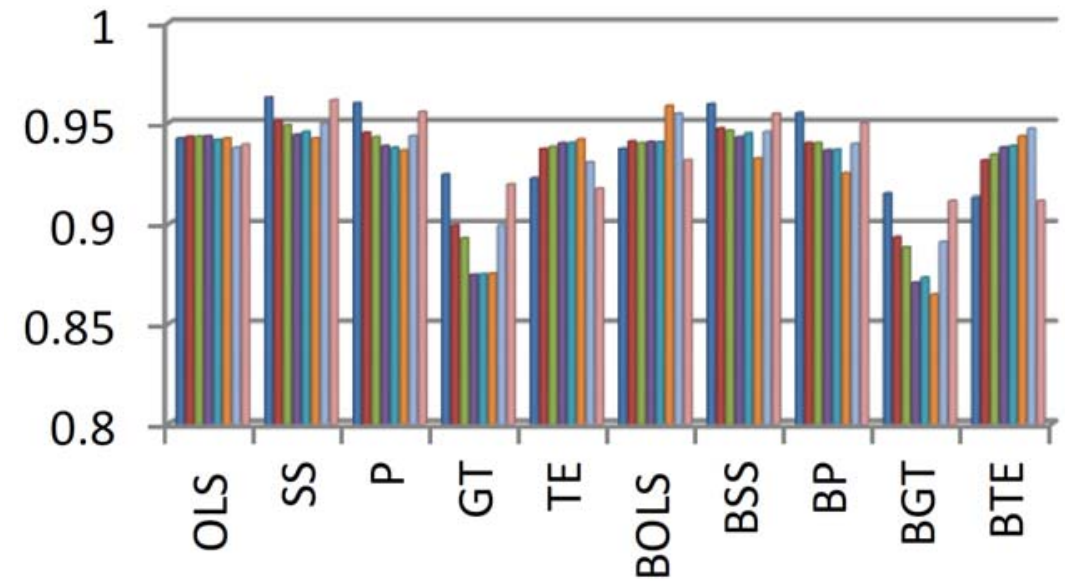

Figure 3.1(a). Coverage probabilities for selected intervals.

$\begin{array}{llll}\square=-0.9 & \square r=-0.8 & \square r=-0.7 & \square r=-0.5 \\ r=0.5 & \square r=0.6 & \square r=0.8 & \square r=0.9\end{array}$

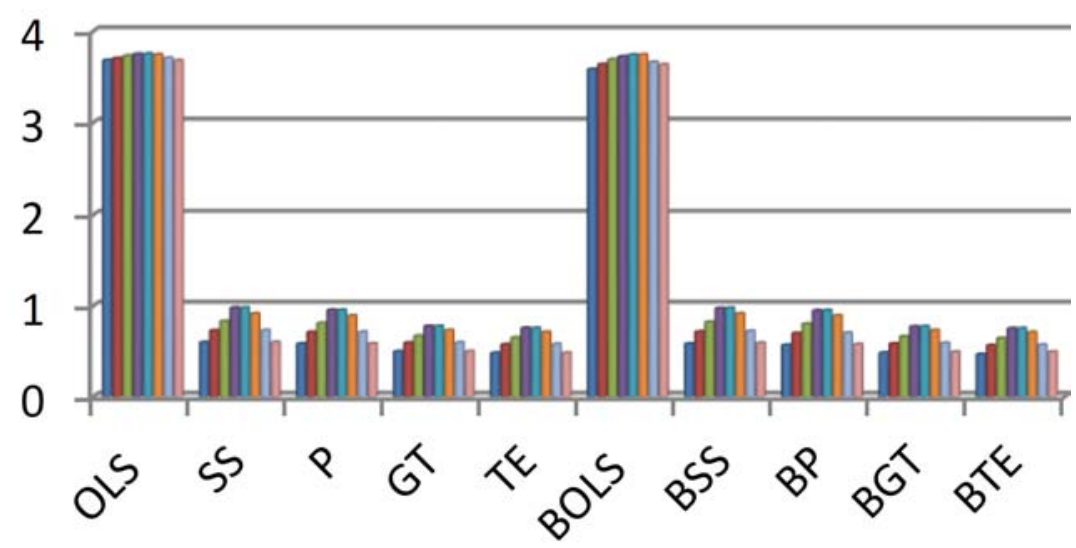

Figure 3.1(b). Mean widths for selected intervals. 
Figure 3.2(a) presents coverage probabilities when data are generated from an $\mathrm{AR}(1)$ model for various values of $\rho$ and moderate sample size $n=50$. We observed from this figure that the So and Shin interval and the Phillips et al. interval have better coverage probabilities as compare to others. It is also observed that our proposed bootstrap versions have better coverage probabilities as compare to selected intervals for construction of confidence intervals for an AR(1) model. Figure 3.2(b) reveals average widths for selected intervals. We found that the Gallagher and Tunno interval and the Tunno and Erwin interval have shorter mean width as compare to other selected intervals. We also notice that our proposed all bootstrap versions have smaller average widths as compare to selected intervals. 
$\square \mathrm{r}=-0.9 \square \mathrm{r}=-0.8 \backsim \mathrm{r}=-0.7 \backsim \mathrm{r}=-0.5$

$\square \mathrm{r}=0.5 \square \mathrm{r}=0.6 \square \mathrm{r}=0.8 \backsim \mathrm{r}=0.9$

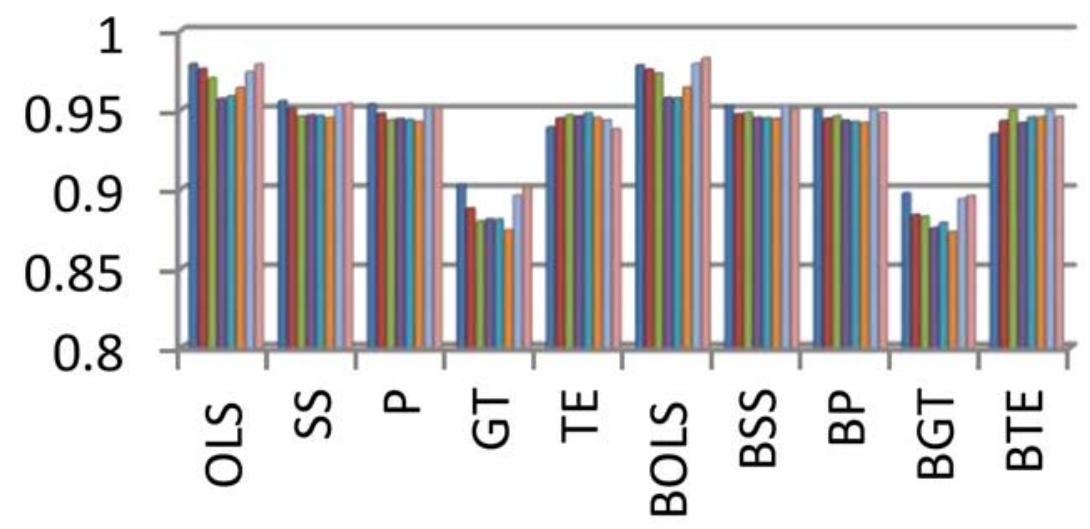

Figure 3.2(a). Coverage probabilities for selected intervals.

$$
\begin{aligned}
& \square=-0.9 \square r=-0.8 \square r=-0.7 \square r=-0.5 \\
& r=0.5 \square r=0.6 \square r=0.8 \square r=0.9
\end{aligned}
$$

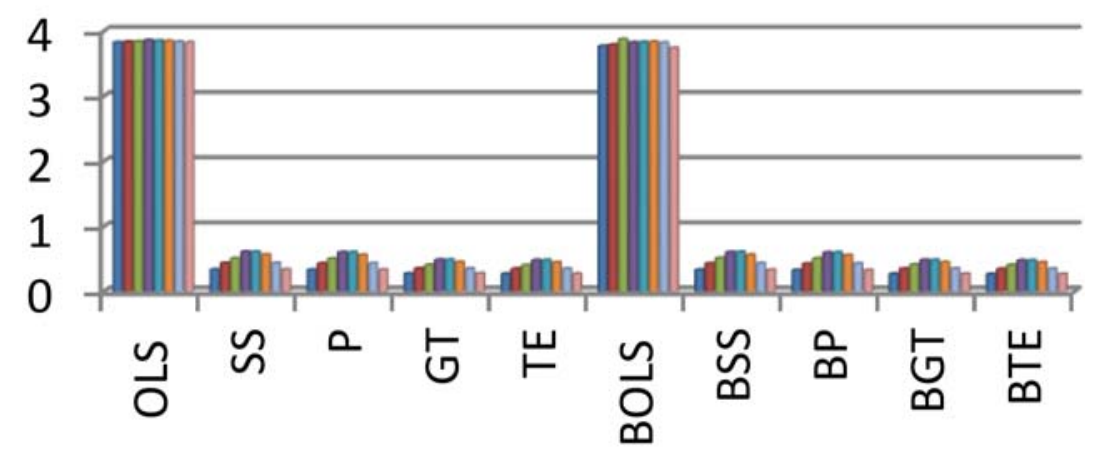

Figure 3.2(b). Mean widths for selected intervals. 
In the Table 3.3, we have reported coverage probabilities and mean widths when data are generated for the large sample size $n=100$ and different values of $\rho$. In the Figure 3.3(a), we have presented coverage probabilities and in the Figure 3.3(b), we have presented the average widths. From the Figure 3.3(a), we observed that all intervals coverage probabilities are very close to the nominal value 0.95 except the Fuller interval, the Gallagher and Tunno interval, bootstrap versions of the Fuller interval and the Gallagher and Tunno interval. But it is noted that all our proposed bootstrap versions coverage probabilities are close to the nominal level as compare to all non-bootstrap versions. In the Figure 3.3(b), we presented average mean widths of selected intervals. It is clear that our proposed bootstrap versions (except the Fuller bootstrap version interval) have smaller average widths as compare to others. 


$$
\begin{aligned}
& \square r=-0.9 \square r=-0.8 \square r=-0.7 \square r=-0.5 \\
& \square r=0.5 \square r=0.6 \square r=0.8 \backsim r=0.9
\end{aligned}
$$

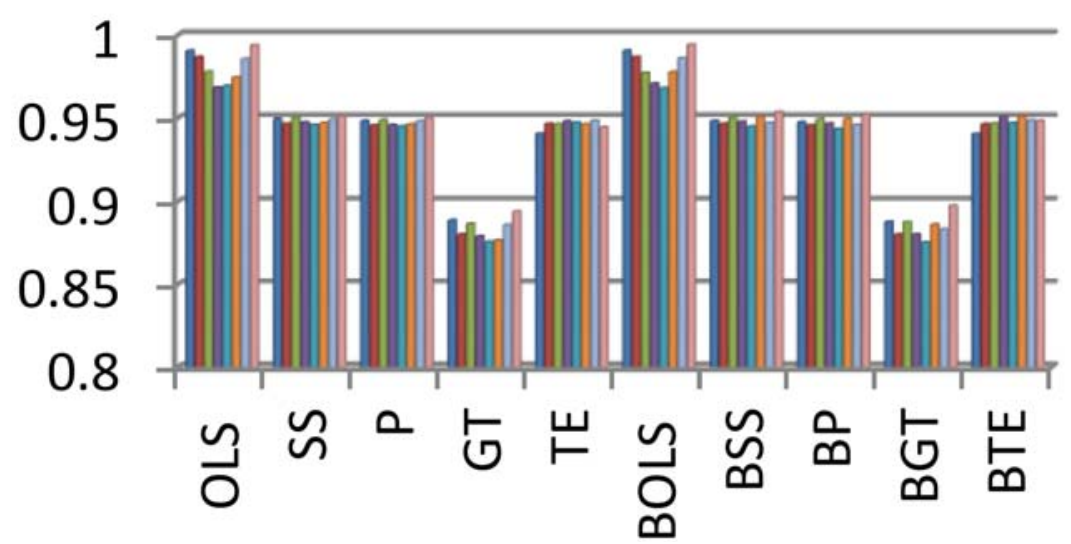

Figure 3.3(a). Coverage probabilities for selected intervals.

$$
\begin{aligned}
& \square r=-0.9 \square r=-0.8 \backsim r=-0.7 \square r=-0.5 \\
& \square r=0.5 \square r=0.6 \square r=0.8 \backsim r=0.9
\end{aligned}
$$

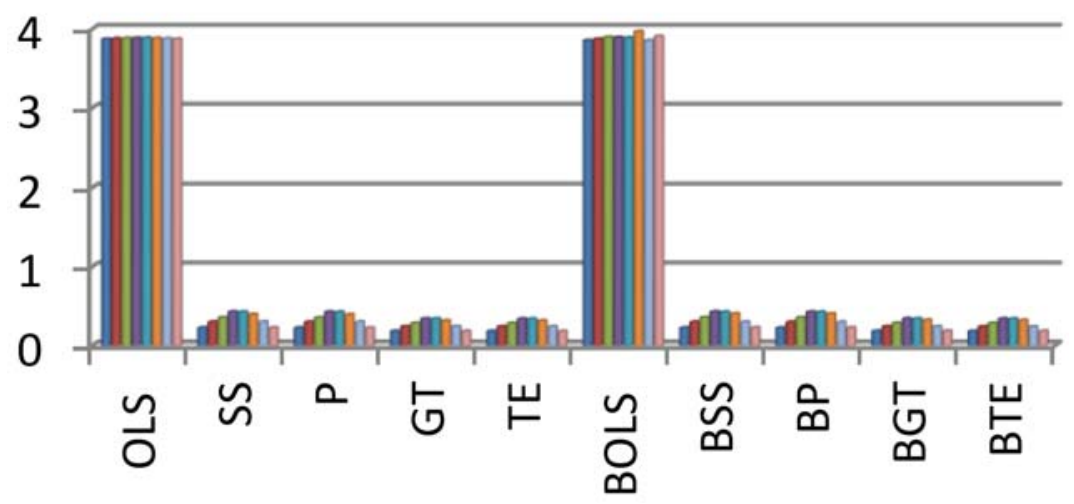

Figure 3.3(b). Average width for selected intervals. 


\section{Application}

In this section, we consider a real-life example to demonstrate the performance of our selected confidence intervals. The data set used is the time series data namely Dhaka daily general stock price index closing values (Source: https://www.dsebd.org) for the period of March 3, 2003 to May 31, 2007. The Dhaka daily general stock price index is a weighted index accounting for companies across major industry groups such as fuel and power, insurance, textiles, bank, cement, engineering, tannery, pharmaceuticals and chemicals, industries and others. The data set is visualized in the Figure 4.1 to understand movement with respect to time. It is observable prices smoothly increased up to 2005 then some volatile patterns for next two years (appropriate reasons of this pattern, refer the newsletters are published by the Dhaka Stock Exchange).

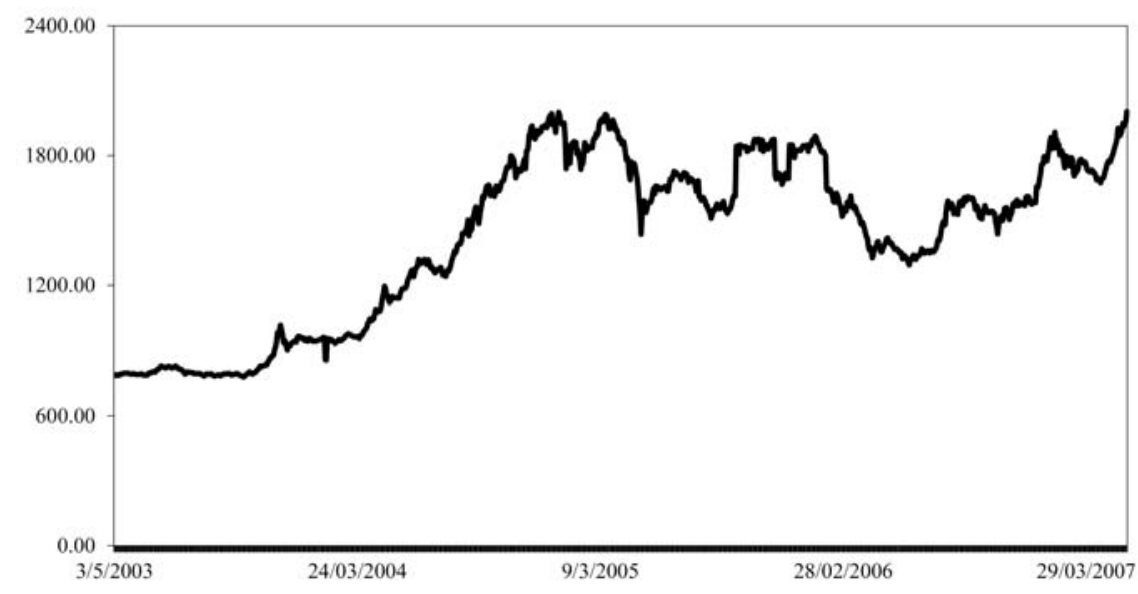

Figure 4.1. Time plots of Dhaka daily general stock price index closing values.

We want to find the $95 \%$ confidence intervals of the parameter of an AR(1) model for the daily stock price of Dhaka Stock Exchange. Findings of this section may be important for stock investors who are interested to know different appearances of stock market to increase their investment 
performances, policy makers who would like to focus to make sensible financial decisions, applied researchers who want to improve the model specifications of this index and others. We observed average daily stock price is 1427.37 and standard deviation is 382.55 meaning considered prices under our study are ranging 1044.82 to 1809.92 .

Since we are interested to find confidence interval of an $\operatorname{AR}(1)$ parameter, that's why we need to know whether our considered data set follows an AR(1) model or not. For this in the following Figure 4.2 we have plotted autocorrelation with respect to various lags (known as correlogram). Figure 4.2 confirms us a pattern of an AR(1) model.

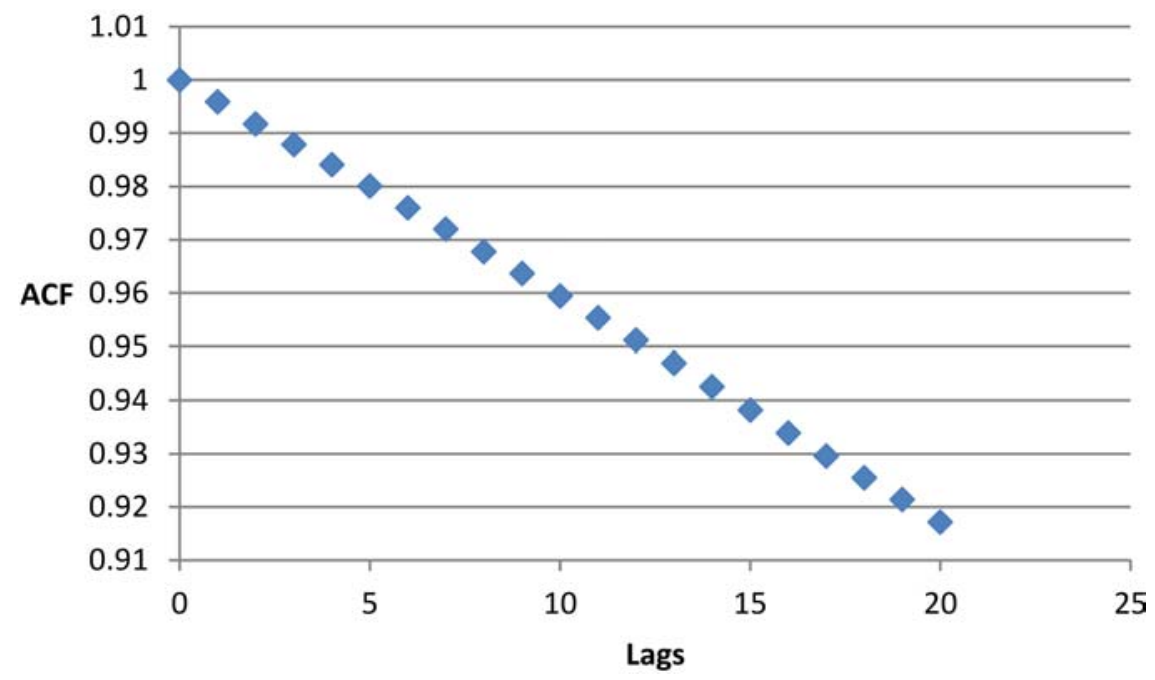

Figure 4.2. Correlogram for an $\mathrm{AR}(1)$ process of Dhaka stock price index values.

The 95\% confidence interval and it's corresponding width of an AR(1) parameter are tabulated in the Table 4.1 and for better understanding in the Figure 4.3. 
Table 4.1. 95\% confidence interval and it's corresponding width of selected intervals

\begin{tabular}{l|c|c}
\hline Intervals & Confidence interval & Width \\
\hline Fuller & $(0.7597,1.2403)$ & 0.4807 \\
So and Shin & $(0.9824,1.0149)$ & 0.0325 \\
Phillips et al. & $(-5.2242,7.2215)$ & 12.4456 \\
Gallagher and Tunno & $(0.9879,1.0123)$ & 0.0244 \\
Tunno and Erwin & $(0.9853,1.0157)$ & 0.0304 \\
Bootstrap OLS & $(0.7856,1.2285)$ & 0.4429 \\
Bootstrap So and Shin & $(0.9841,1.0141)$ & 0.0300 \\
Bootstrap Phillips et al. & $(-4.5528,6.9148)$ & 11.4677 \\
Bootstrap Gallagher and Tunno & $(0.9946,1.0136)$ & 0.0190 \\
Bootstrap Tunno and Erwin & $(0.9860,1.0140)$ & 0.0280 \\
\hline
\end{tabular}

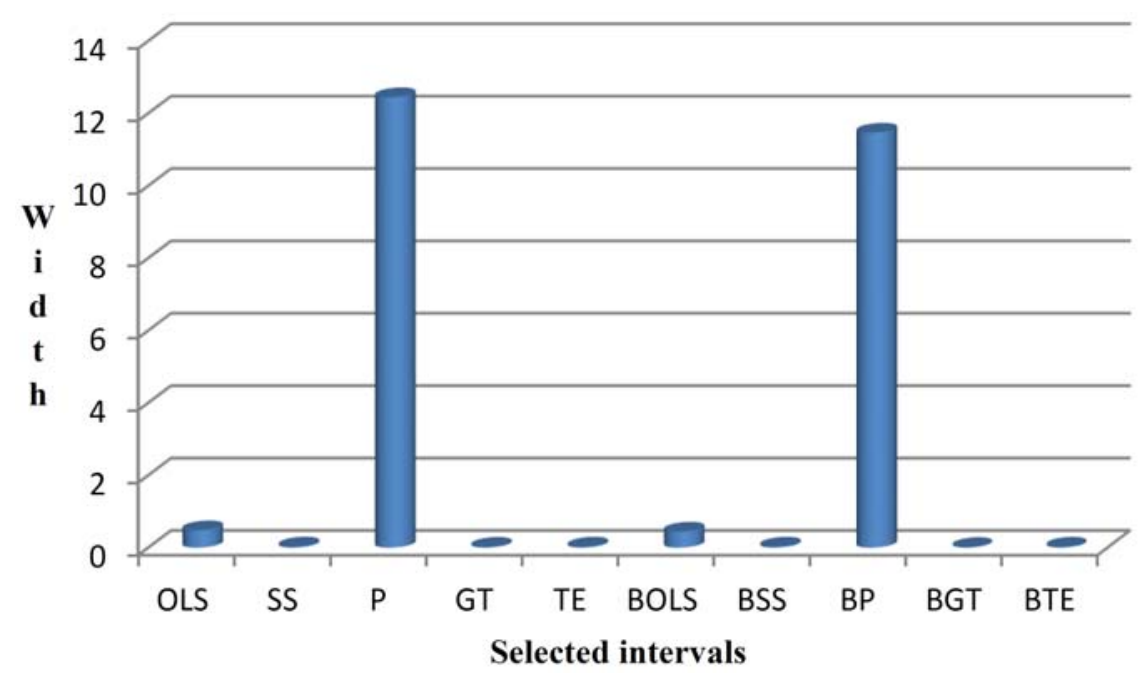

Figure 4.3. Width for an $\mathrm{AR}(1)$ process of Dhaka stock price index values.

We noted that the bootstrap version of the Gallagher and Tunno interval has the smallest width followed by the Gallagher and Tunno interval, the bootstrap version of Tunno and Erwin interval, the bootstrap version of So and Shin interval, the Tunno and Erwin interval, 
the So and Shin interval, the bootstrap version of the Fuller interval, the Fuller interval, the bootstrap version of Philips et al. interval and the Philips et al. interval. Note that the Philips et al. interval has the highest width as compare to other intervals.

\section{Conclusion}

This paper proposes some bootstrap versions confidence intervals that are available in literature for estimating the parameter of the autoregressive time series model of order 1 . To compare the performance of the intervals estimators, a simulation study has been done. Data were generated from an autoregressive time series model of order 1 and 95\% coverage probability and average width are calculated for all intervals. Our observation from the simulation study is that all proposed intervals have coverage probabilities closest to the nominal level. However, the average widths for all proposed bootstrap versions have lower average widths as compare to the existing intervals. A real life application has been given to illustrate the simulation findings of the paper.

\section{References}

[1] Beong Soo So and Dong Wan Shin, Cauchy estimators for autoregressive processes with applications to unit root tests and confidence intervals, Econometric Theory 15(2) (1999), 165-176.

[2] D. A. Dickey and W. A. Fuller, Distribution of the estimators for autoregressive time series with a unit root, Journal of the American Statistical Association 74(366) (1979), 427-431.

DOI: https://doi.org/10.1080/01621459.1979.10482531

[3] B. Efron, Bootstrap methods: Another look at the jackknife, Annals of Statistics 7(1) (1979), 1-26.

DOI: https://doi.org/10.1214/aos/1176344552

[4] F. Tunno and A. Erwin, New confidence intervals for the AR(1) parameter, Involve 6(1) (2013), 53-63.

DOI: https://doi.org/10.2140/involve.2013.6.53

[5] W. A. Fuller, Introduction to Statistical Time Series, Wiley, New York, 1976. 
[6] C. Gallagher and F. Tunno, A small sample confidence interval for autoregressive parameters, Journal of Statistical Planning and Inference 138(12) (2008), 3858-3868.

DOI: https://doi.org/10.1016/j.jspi.2008.02.001

[7] P. C. B. Phillips, J. Y. Park and Y. Chang, Nonlinear instrumental variable estimation of an autoregression, Journal of Econometrics 118(1-2) (2004), 219-246.

DOI: https://doi.org/10.1016/S0304-4076(03)00141-6 